\title{
Hyperoncotic Albumin Solution in Continuous Renal Replacement Therapy Patients
}

\author{
Zachary O'Brien a, b, c Mark Finnis ${ }^{c, d, e}$ Martin Gallagher ${ }^{f}$ Rinaldo Bellomo $^{\text {a, c, e, g }}$ \\ on behalf of the RENAL Study Investigators and the Australian and New Zealand \\ Intensive Care Clinical Trials Group
}

${ }^{a}$ Department of Intensive Care, Austin Hospital, Melbourne, VIC, Australia; ${ }^{b}$ Department of Anaesthesia, Austin Hospital, Melbourne, VIC, Australia; 'Australian and New Zealand Intensive Care Research Centre, School of Public Health and Preventive Medicine, Monash University, Melbourne, VIC, Australia; ${ }^{\mathrm{d} D e p a r t m e n t}$ of Intensive Care, Royal Adelaide Hospital, Adelaide, SA, Australia; 'Department of Critical Care, School of Medicine, The University of Melbourne, Melbourne, VIC, Australia; ${ }^{\top}$ The George Institute for Global Health, University of New South Wales, Sydney, NSW, Australia; ${ }^{9}$ Data Analytics Research and Evaluation (DARE) Centre, The University of Melbourne and Austin Hospital, Melbourne, VIC, Australia

\section{Keywords}

Hyperoncotic albumin · Renal replacement therapy · Acute kidney injury

\begin{abstract}
Aim: The aim of this study was to investigate the association of hyperoncotic (20\%) human albumin solution (HAS) with outcomes among critically ill patients receiving continuous renal replacement therapy (RRT). Methods: Analysis of the Randomized Evaluation of Normal versus Augmented Level (RENAL) RRT trial data. Results: Of 1,508 patients, 771 (51\%) received albumin. Of these, 345 (45\%) received $4 \%$ HAS only, 155 (20\%) received 20\% HAS only, and 271 (35\%) received both. Patients who received combined 4\% and 20\% HAS were more severely ill, received more days of RENAL trial therapy and required mechanical ventilation for longer. Mean daily fluid balance was $-288 \mathrm{~mL}$ (-904 to 261) with $20 \%$ HAS only versus $245 \mathrm{~mL}(-248$ to 1,050$)$ with $4 \%$ HAS only ( $p<0.001$ ). On Cox proportional hazards regression, $20 \%$ HAS exposure was not associated with greater 90 -day
\end{abstract}

karger@karger.com www.karger.com/bpu

Karger $\stackrel{\text { ' }}{5}$

BOPEN ACCESS
(C) 2021 The Author(s)

Published by S. Karger AG, Basel

This is an Open Access article licensed under the Creative Commons Attribution-NonCommercial-4.0 International License (CC BY-NC) (http://www.karger.com/Services/OpenAccessLicense), applicable to the online version of the article only. Usage and distribution for commercial purposes requires written permission. mortality (odds ratio 1.12, 95\% confidence interval [CI]: $0.77-$ $1.62 ; p=0.55$ ) or longer recovery to RRT independence (subhazard ratio $1.04,95 \% \mathrm{Cl}: 0.84-1.30 ; p=0.70)$ compared to those who received $4 \%$ HAS only. Conclusions: RENAL trial patients commonly received albumin in varying concentrations. The administration of $20 \%$ HAS was associated with a more negative fluid balance but was not independently associated with increased mortality or RRT dependence when compared to $4 \%$ HAS only.
(C) 2021 The Author(s)

Published by S. Karger AG, Basel

\section{Introduction}

Intravenous (IV) fluids are frequently administered to patients in intensive care units (ICUs) [1,2]. The selection of such fluids should involve the same considerations as any other medication $[3,4]$. A limited number of fluids are

A complete list of investigators in the RENAL study is provided in Supplemental Digital Content 1 (Item S1)

Correspondence to:

Rinaldo Bellomo, rinaldo.bellomo@ austin.org.au 
in common usage however, with varying impacts on patient outcomes [5-9]. Of particular concern is the potential for nephrotoxic effects of some fluid types [5-7, 10-12], with multiple studies demonstrating an association between artificial colloids and worsening acute kidney injury (AKI) $[7,10,11]$. Therefore, when indicated, human albumin solution (HAS) may be the colloid fluid of choice in patients with or at risk of severe AKI. Accordingly, iso-oncotic (4 or 5\%) HAS has been used during high-volume resuscitation in critically ill patients and during renal replacement therapy (RRT) to attenuate hypotension and/or facilitate ultrafiltration $[13,14]$. In contrast, there is essentially no data on the independent association of hyperoncotic (20\%) HAS with clinical outcomes when used during RRT. In particular, iso-oncotic and hyperoncotic HAS are yet to be directly compared in ICU patients receiving RRT for severe AKI $[12,15,16]$. This is despite concern that hyperoncotic solutions may decrease the glomerular filtration rate [14]. Therefore, a direct comparison of iso-oncotic HAS to hyperoncotic HAS is required, to determine whether the formulation of HAS mediates an effect on renal outcomes, as opposed to an effect intrinsic to albumin itself.

The Randomized Evaluation of Normal versus Augmented Level (RENAL) replacement therapy study enrolled a large cohort of critically ill patients treated with continuous RRT for severe AKI in Australia and New Zealand [17]. The study protocol included obtaining detailed data on the administration of HAS. Accordingly, we aimed to use the RENAL database to investigate the association between exposure to hyperoncotic $20 \%$ HAS and mortality, recovery to RRT independence, and other clinical outcomes.

\section{Materials and Methods}

The RENAL study was a multicentre, prospective, randomized trial comparing two levels of intensity of continuous RRT in 1,508 adult ( $>18$ years) critically ill patients with AKI, conducted in 35 ICUs in Australia and New Zealand [17, 18]. The Human Research Ethics Committees of the University of Sydney and all participating institutions approved the study (GI-RE-ARF001-40-R). The features of the trial have been previously described [17, 18]. Patients enrolled had AKI requiring RRT and met at least one of the following criteria: oliguria unresponsive to fluid resuscitation, hyperkalaemia, severe academia, blood urea nitrogen $>70 \mathrm{mg} / \mathrm{dL}$ (>25 mmol/L), creatinine $>3.4 \mathrm{mg} / \mathrm{dL}(>300 \mu \mathrm{mol} / \mathrm{L})$, or severe organ oedema (clinically significant organ oedema in the setting of acute renal failure, such as pulmonary oedema, as determined by the treating clinician). Patients were treated with continuous veno-venous hemodiafiltration using post-dilution and were randomly assigned to either a higher intensity group (effluent flow rate $40 \mathrm{~mL} / \mathrm{kg} / \mathrm{h}$ ) or lower intensity group (effluent flow rate 25
$\mathrm{mL} / \mathrm{kg} / \mathrm{h}$ ). The primary study outcome was all-cause mortality within 90 days of randomization (secondary outcomes are outlined in online suppl.; for all online suppl. material, see www. karger.com/doi/10.1159/000517957). Further details are available in the original RENAL study publication $[17,18]$.

\section{HAS Therapy}

We studied the subgroup of trial patients who received HAS at any stage within the study period. The decision to administer albumin was at the discretion of the treating clinician. The volumes of $4 \%$ and/or $20 \%$ HAS administered to patients were prospectively collected daily, until death, ICU discharge, or day 28 postrandomization (whichever occurred first). Serum albumin levels were measured daily on morning blood tests for the same period.

\section{Statistical Analysis}

Continuous variables were expressed as mean \pm SD or median (interquartile range) and compared using Student's $t$ test or the Wilcoxon rank-sum test, as appropriate. Categorical variables were expressed as number (percent) and compared using Pearson's $\chi^{2}$ or Fisher's exact test.

We studied the association between HAS concentration and 90day mortality using a variety of regression techniques. We performed univariate and multivariable logistic regression adjusting for baseline variables which were clinically or statistically significant, with robust standard errors adjusted for clustering by the ICU site. We created a propensity score model consisting of those variables which were strongly associated with either 90-day mortality or the administration of $20 \%$ albumin, employing a backward stepwise elimination process $(p<0.1)$. We used this propensity score model to estimate the average treatment effect using stabilized inverse probability of treatment weighting (SIPW), as has been utilized in previous observational trials and post hoc analyses [19-22]. Specifically, we defined the propensity score as the probability of a patient being selected for a specific treatment (e.g., 20\% albumin) conditional upon available baseline characteristics. Weighting patients by the inverse probability of treatment creates a pseudo-population in which treatment is independent of measured baseline covariates. Stabilized weights reduce the influence of patients with either very low or very high propensity scores [23]. Data were analysed using STATA version 16.0 (Stata, College Station, TX, USA).

We presented time to death within 90 days as Kaplan-Meier curves and compared using Cox proportional hazards regression, with and without SIPW adjustment. We presented results as odds ratios with $95 \%$ confidence intervals (95\% CIs) and associated $p$ values. The durations of RRT, mechanical ventilation (MV), and ICU and hospital stay were studied using competing risks regression, with death as the competing event, both with and without SIPW adjustment, and are presented as sub-hazard ratios (SHRs) with associated $95 \%$ CIs and $p$ values. $p$ values are provided for perspective rather than dichotomous "statistical significance" testing, given all findings are exploratory.

\section{Results}

\section{Patients}

The screening, enrolment, and randomization of patients in the RENAL trial is presented in the original trial 
Table 1. Baseline patient characteristics of recipients of 4 versus $20 \%$ versus combined $(4+20 \%)$ albumin

\begin{tabular}{|c|c|c|c|c|}
\hline Characteristic & $\begin{array}{l}\text { Albumin } \\
4 \% \text { only }\end{array}$ & $\begin{array}{l}\text { Albumin } \\
20 \% \text { only }\end{array}$ & $\begin{array}{l}\text { Combined } \\
4 \text { and } 20 \%\end{array}$ & $p$ value \\
\hline Number (unless indicated, $n$ ) & $345(45)$ & $155(20)$ & $271(35)$ & \\
\hline Age, mean (SE), years & $66(13.7)$ & $64(14.0)$ & $63(15.0)$ & 0.14 \\
\hline Male gender, $n(\%)$ & $238(69)$ & $111(72)$ & $169(63)$ & 0.09 \\
\hline Weight, mean (SD), kg & $81(13.1)$ & $81(12.8)$ & $81(13.1)$ & 0.95 \\
\hline APACHE III score, mean $(\mathrm{SD})^{\S}$ & $103(28)$ & $100(23)$ & $103(25)$ & 0.54 \\
\hline Creatinine pre-randomization $(\mathrm{SD})^{\dagger}, \mu \mathrm{mol} / \mathrm{L}$ & $313(172)$ & $315(173)$ & $282(145)$ & 0.03 \\
\hline eGFR, mean $(\mathrm{SD})(n=81)$ & $58(33)$ & $64(32)$ & $59(26)$ & 0.30 \\
\hline eGFR group, $n(\%)$ & $n=196$ & $n=90$ & $n=139$ & \\
\hline$<30 \mathrm{~mL} / \mathrm{min}$ & $39(20)$ & $11(12)$ & $17(12)$ & \\
\hline 30 to $<46 \mathrm{~mL} / \mathrm{min}$ & $38(19)$ & $15(17)$ & $25(18)$ & 0.42 \\
\hline 46 to $<60 \mathrm{~mL} / \mathrm{min}$ & $37(19)$ & $18(20)$ & $31(22)$ & \\
\hline$\geq 60 \mathrm{~mL} / \mathrm{min}$ & $82(42)$ & $46(51)$ & $66(47)$ & \\
\hline $\mathrm{BUN}$, mean $(\mathrm{SD})^{\dagger \dagger}, \mathrm{mmol} / \mathrm{L}$ & $23(12.7)$ & $24(12.3)$ & $22(11.6)$ & 0.22 \\
\hline Plasma albumin, mean (SD), g/L & $27(7.3)$ & $24(6.4)$ & $24(7.1)$ & $<0.001$ \\
\hline $\mathrm{pH}$, mean $(\mathrm{SD})$ & $7.25(0.14)$ & $7.31(0.11)$ & $7.27(0.12)$ & $<0.001$ \\
\hline Bicarbonate, mean (SD), $\mathrm{mmol} / \mathrm{L}$ & $18.2(6.5)$ & $20.3(5.6)$ & $18.6(5.5)$ & 0.001 \\
\hline Base excess, mean (SD), mmol/L & $-8.4(7.4)$ & $-5.7(6.5)$ & $-7.7(6.6)$ & $<0.001$ \\
\hline Mechanical ventilation, $n(\%)$ & $275(80)$ & $115(74)$ & $221(82)$ & 0.19 \\
\hline \multicolumn{5}{|l|}{ SOFA score, mean (SD) } \\
\hline Cardiovascular, $N=769$ & $3.0(1.4)$ & $2.9(1.5)$ & $3.3(1.3)$ & 0.02 \\
\hline Respiratory, $N=755$ & $2.9(0.9)$ & $2.7(1.0)$ & $2.9(0.9)$ & 0.04 \\
\hline Coagulation, $N=767$ & $1.0(1.1)$ & $1.1(1.1)$ & $1.1(1.3)$ & 0.60 \\
\hline Liver, $N=738$ & $0.9(1.0)$ & $1.3(1.3)$ & $1.2(1.2)$ & $<0.001$ \\
\hline \multicolumn{5}{|l|}{ Source of admission } \\
\hline Emergency department & $85(25)$ & $28(18)$ & $53(20)$ & \\
\hline Hospital ward & $87(25)$ & $46(30)$ & $63(23)$ & \\
\hline Transfer from another ICU & $33(9.6)$ & $11(7.1)$ & $28(10)$ & \\
\hline Transfer from another hospital & $32(9.3)$ & $12(7.7)$ & $22(8.1)$ & 0.12 \\
\hline OR after emergency surgery & $51(15)$ & $18(12)$ & $49(18)$ & \\
\hline OR after elective surgery & $37(11)$ & $19(12)$ & $36(13)$ & \\
\hline Unknown & $20(5.8)$ & $21(14)$ & $20(7.4)$ & \\
\hline Severe sepsis, $n(\%)$ & $165(48)$ & $93(60)$ & $159(59)$ & 0.01 \\
\hline \multicolumn{5}{|l|}{ Admission diagnostic group, $n(\%)$} \\
\hline Non-operative & $236(68)$ & $112(72)$ & $174(64)$ & \\
\hline Cardiovascular & $131(38)$ & $61(39)$ & $101(37)$ & \\
\hline Genitourinary & $38(11)$ & $22(14)$ & $27(10)$ & \\
\hline Respiratory & $36(10)$ & $18(12)$ & $26(9.6)$ & \\
\hline Gastrointestinal & $14(4.1)$ & $7(4.5)$ & $11(4.1)$ & \\
\hline Other & $17(4.9)$ & $4(2.6)$ & $9(3.3)$ & 0.32 \\
\hline Operative & $109(32)$ & $43(28)$ & $97(36)$ & \\
\hline Cardiovascular & $77(22)$ & $27(17)$ & $55(20)$ & \\
\hline Gastrointestinal & $24(7.0)$ & $10(6.5)$ & $31(11)$ & \\
\hline Trauma & $3(0.9)$ & 0 & $6(2.2)$ & \\
\hline Other & $5(1.4)$ & $6(3.9)$ & $5(1.8)$ & \\
\hline \multicolumn{5}{|l|}{ Criteria for randomization $\diamond$} \\
\hline Oliguria $(<400$ mL/day) & $221(64)$ & $80(52)$ & $139(51)$ & 0.002 \\
\hline Hyperkalaemia & $25(7.3)$ & $8(5.2)$ & $12(4.4)$ & 0.31 \\
\hline Severe acidaemia & $128(37)$ & $36(23)$ & $88(32)$ & 0.01 \\
\hline $\begin{array}{l}\mathrm{BUN}>70 \mathrm{mg} / \mathrm{dL} \\
\quad(\text { Urea }>25 \mathrm{mmol} / \mathrm{L})\end{array}$ & $129(37)$ & $68(44)$ & $109(40)$ & 0.38 \\
\hline $\begin{array}{l}\text { Creatinine }>3.4 \mathrm{mg} / \mathrm{dL} \\
\quad(300 \mu \mathrm{mol} / \mathrm{L})\end{array}$ & $152(44)$ & $68(44)$ & $99(37)$ & 0.13 \\
\hline Severe organ oedema with AKI & $134(39)$ & $82(53)$ & $150(55)$ & $<0.001$ \\
\hline Randomized to high intensity continuous RRT, $n(\%)$ & $175(50.7)$ & $75(48.4)$ & $136(50.2)$ & 0.89 \\
\hline
\end{tabular}


Table 1 (Footnote)

APACHE, Acute Physiology Assessment and Chronic Health Evaluation; BUN, blood urea nitrogen; eGFR, estimated glomerular filtration rate; OR, odds ratio; SOFA, sequential organ failure assessment; RRT, renal replacement therapy; AKI, acute kidney injury; ICU, intensive care unit. ${ }^{\S}$ APACHE III scores range from 0 to 299 , with higher scores indicating more severe illness. ${ }^{\dagger}$ To convert the values for creatinine to milligrams per decilitre, divide by 88.4. ${ }^{\dagger \dagger}$ To convert the values for BUN to milligrams per decilitre, divide by 0.357. 9 SOFA scores range from 0 to 4 , with a higher score indicating more severe organ dysfunction. ${ }^{\diamond} \mathrm{A}$ given patient may have met more than 1 of these criteria.

publication. Of the 1,508 patients included in the RENAL trial, 771 (51\%) received HAS. Albumin was administered exclusively in the form of $4 \%$ HAS to 345 (45\%) patients, as 20\% HAS in isolation to $155(20 \%)$ patients, and as a combination to $271(35 \%)$ patients. Several statistically significant differences existed between these groups at baseline (Table 1).

A greater proportion of patients who received $20 \%$ HAS had severe organ oedema, while a greater proportion of patients who received $4 \%$ HAS had oliguria and severe acidaemia. Those who received $20 \%$ HAS in combination with $4 \%$ HAS had lower serum albumin and creatinine levels at baseline and higher Sequential Organ Failure Assessment cardiovascular and liver scores (Table 1).

\section{Process of Care}

Patients who received $4 \%, 20 \%$, or both received 8,10 , and 17 days of RENAL trial therapy, respectively $(p<$ 0.001 ) (Table 2). The number of patients receiving HAS decreased after day 2 (online suppl. Fig. 1, 2 in Supplementary Material 1).

Among patients who received both $4 \%$ and $20 \%$ HAS, a greater proportion received $4 \%$ HAS on days 1 and 2, though beyond this point the ratio of $4-20 \%$ was similar for the ongoing duration of treatment (online suppl. Fig. 1,2 ). Patients who received $20 \%$ HAS, exclusively or in combination with $4 \%$ HAS, had slightly lower plasma albumin throughout the study period. Patients who received both also received substantially more albumin overall (10.5 g [6.5-19.9] per study day) than 4 or $20 \%$ HAS in isolation (5.0 g [2.2-10.0] and $5.7 \mathrm{~g}$ [3.3-12.5] per study day) $(p<0.001)$.

A greater proportion of patients who received combined 4 and 20\% HAS also received fresh frozen plasma, packed red blood cells, platelets, cryoprecipitate and enteral or parental nutrition, and at greater volumes per study day, than those who received either 4 or $20 \%$ albumin alone (Table 2).

Variation in the concentration of HAS administered was associated with only small differences in the volume of other IV fluids administered, with an average daily volume of 1.6 L (1.2-2.1 L), 1.4 L (1.0-1.7 L), and 1.6 L (1.2$2.0 \mathrm{~L}$ ) of crystalloid given to patients administered $4 \%$, $20 \%$, and combined HAS, respectively $(p<0.001)$. However, administration of $20 \%$ HAS only resulted in a mean daily fluid balance of minus $288 \mathrm{~mL}$ ( -904 to 261 ), while a positive daily fluid balance was recorded with $4 \%$ HAS only $(245 \mathrm{~mL}[-248$ to 1,050$])$ or combined HAS $(88 \mathrm{~mL}$ [-352 to 608$])(p<0.001)$.

\section{Primary Outcome}

Overall, 167 (48\%) 4\% HAS patients, 80 (52\%) 20\% HAS patients, and 139 (51\%) combined HAS patients died within 90 days of randomization $(p=0.65)$ (Table 3 ). On SIPW-adjusted Cox proportional hazards analysis, the administration of $20 \%$ HAS (either alone or in combination) was not associated with 90-day mortality (odds ratio $=1.12$, 95\% CI: 0.77-1.62; $p=0.55)($ Table 4; Fig. 1a) .

\section{Renal Secondary Outcomes}

Patients who received a combination of $4 \%$ and $20 \%$ HAS underwent RRT for a longer duration than those who received $4 \%$ or $20 \%$ HAS alone ( 9 vs. 4 vs. 6 days, respectively, $p<0.001$ ) (Table 3 ). The proportion of patients who remained dependent on RRT at 28 and 90 days was similar between groups (Table 3 ). There was no independent association between $20 \%$ HAS therapy and recovery to RRT independence after SIPW modelling $(\mathrm{SHR}=1.04,95 \%$ CI $0.84-1.30 ; p=0.70)$ (Table 4; Fig. 1b).

\section{Tertiary Outcomes}

Patients who received combined $4 \%$ and $20 \%$ HAS were mechanically ventilated for an average of 12 days, compared to 6 days in those who received either $4 \%$ or $20 \%$ alone $(p<0.001)$ and had an increased dependence on MV at 28 days (14.7 vs. 6.1 vs. $4.6 \% ; p=0.007$ ) (Table 3). On SIPW analysis, the administration of $20 \%$ HAS was not independently associated with the duration of MV ( $p=0.37)$ (Table 4; Fig. 1c). On multivariate SIPW analysis, the administration of $20 \%$ HAS was not associ- 
Table 2. Process of care of recipients of $4 \%$ versus $20 \%$ versus combined $(4+20 \%)$ albumin

\begin{tabular}{|c|c|c|c|c|}
\hline Characteristic & $\begin{array}{l}\text { Albumin } \\
4 \% \text { only }\end{array}$ & $\begin{array}{l}\text { Albumin } \\
20 \% \text { only }\end{array}$ & $\begin{array}{l}\text { Combined } \\
4 \% \text { and } 20 \%\end{array}$ & $p$ value \\
\hline Number (unless indicated, $n$ ) & $345(45)$ & $155(20)$ & $271(35)$ & \\
\hline Study Days of care, median (IQR) & $8[4,14]$ & $10[6,16]$ & $17[10,26]$ & $<0.001$ \\
\hline \multicolumn{5}{|l|}{ Plasma albumin, mean (SD), g/L } \\
\hline Baseline & $27.3(7.3)$ & $24.3(6.4)$ & $24.4(7.1)$ & $<0.001$ \\
\hline Average & $26.0(5.3)$ & $24.8(5.5)$ & $24.5(5.1)$ & 0.001 \\
\hline Minimum & $22.4(6.0)$ & $20.6(5.9)$ & $19.0(5.3)$ & $<0.001$ \\
\hline \multicolumn{5}{|l|}{ Albumin, grams, median (IQR) } \\
\hline Total grams & $40[20,72]$ & $40[39,100]$ & $149[90,264]$ & $<0.001$ \\
\hline Mean grams per study day & $5.0[2.2,10.0]$ & $5.7[3.3,12.5]$ & $10.5[6.5,19.9]$ & $<0.001$ \\
\hline Fresh frozen plasma, $n(\%)$ & $121(35)$ & $39(25)$ & $132(49)$ & $<0.001$ \\
\hline Total mL, median (IQR) & $1,000[563,2,128]$ & $2,094[671,3,240]$ & $1,672[868,2,898]$ & 0.001 \\
\hline Total grams-albumin $\diamond$ & $33[19,70]$ & $69[22,107]$ & $55[29,96]$ & 0.001 \\
\hline Mean grams-albumin per day $\diamond$ & $4.7[1.7,11.4]$ & $5.4[2.8,9.9]$ & $3.1[1.4,7.8]$ & 0.055 \\
\hline Red blood cells, $n(\%)$ & $245(71)$ & $120(77)$ & $246(91)$ & $<0.001$ \\
\hline Total mL, median (IQR) & $598[0,1,361]$ & $795[253,1,618]$ & $1,770[808,3,494]$ & $<0.001$ \\
\hline Mean mL/day & $199[0,322]$ & $205[75,308]$ & $241[143,357]$ & 0.002 \\
\hline \multicolumn{5}{|l|}{ Other blood products, $n(\%)$} \\
\hline Platelets & $101(29)$ & $43(28)$ & $139(51)$ & $<0.001$ \\
\hline Cryoprecipitate & $29(8.4)$ & $10(6.5)$ & $50(19)$ & $<0.001$ \\
\hline \multicolumn{5}{|l|}{ Enteral nutrition } \\
\hline Number $(\%)$ & $272(79)$ & $123(79)$ & $240(89)$ & 0.004 \\
\hline Mean mL/day, median (IQR) & $792[486,1,071]$ & $840[584,1,078]$ & $936[638,1,212]$ & 0.002 \\
\hline \multicolumn{5}{|l|}{ Parenteral nutrition } \\
\hline Number $(\%)$ & $136(39)$ & $76(49)$ & $167(62)$ & $<0.001$ \\
\hline Mean mL/day, median (IQR) & $393[94,1,145]$ & $389[83,1,225]$ & $787[113,1,395]$ & 0.10 \\
\hline \multicolumn{5}{|l|}{ CRRT fluid, med (IQR), L } \\
\hline Average L/day & $24.5[14.2,34.9]$ & $23.9[15.6,32.9]$ & $22.8[11.2,37.4]$ & 0.92 \\
\hline Cumulative total & $155[83,321]$ & $203[112,349]$ & $272[147,514]$ & $<0.001$ \\
\hline \multicolumn{5}{|l|}{ Other IV fluid, median (IQR) } \\
\hline Average L/day & $1.6[1.2,2.1]$ & $1.4[1.0,1.7]$ & $1.6[1.2,2.0]$ & $<0.001$ \\
\hline Cumulative total & $13[7,21]$ & $14[7,21]$ & $25[15,40]$ & $<0.001$ \\
\hline \multicolumn{5}{|l|}{ Fluid balance, median (IQR) } \\
\hline Average daily, $\mathrm{mL}$ & $243[-248,1,050]$ & $-288[-904,261]$ & $88[-352,608]$ & $<0.001$ \\
\hline Cumulative total, $\mathrm{L}$ & $1.9[-2.4,6.4]$ & $-2.4[-9.5,1.7]$ & $1.3[-5.3,7.4]$ & $<0.001$ \\
\hline \multicolumn{5}{|l|}{ Urine output, median (IQR) } \\
\hline Average daily, $\mathrm{mL}$ & $556[71,1,670]$ & $741[138,1,404]$ & $934[211,1,817]$ & 0.02 \\
\hline Cumulative total, $\mathrm{L}$ & $4.8[0.5,17.8]$ & $5.5[1.1,16.9]$ & $13.4[1.9,34]$ & $<0.001$ \\
\hline \multicolumn{5}{|l|}{ Total SOFA scores, median (IQR) } \\
\hline Mean of averaged scores & $1.8[1.3,2.4]$ & $1.6[1.3,2.4]$ & $1.8[1.4,2.4]$ & 0.566 \\
\hline Maximum of averaged scores & $2.8[2.2,3.5]$ & $2.7[2.0,3.3]$ & $3.0[2.5,3.5]$ & $<0.001$ \\
\hline Mean of summed scores & $10.0[7.4,13.1]$ & $9.4[7.3,13.0]$ & $10.4[7.7,13.7]$ & 0.179 \\
\hline Maximum of summed scores & $14.4[12.0,16.8]$ & $14.4[10.8,16.8]$ & $15.6[13.2,18.0]$ & $<0.001$ \\
\hline \multicolumn{5}{|l|}{ Cardiovascular SOFA score } \\
\hline Mean score, median (IQR) & $2.1[1.2,3.7]$ & $2.2[1.1,3.5]$ & $2.3[1.5,3.2]$ & 0.44 \\
\hline Maximum score, median (IQR) & $4.0[3.0,4.0]$ & $4.0[3.0,4.0]$ & $4.0[4.0,4.0]$ & 0.001 \\
\hline Maximum score, mean $(\mathrm{SD}) \S$ & $3.5(1.0)$ & $3.5(1.0)$ & $3.9(0.5)$ & $<0.001$ \\
\hline \multicolumn{5}{|l|}{ Respiratory SOFA score } \\
\hline Mean score, median (IQR) & $2.9[2.4,3.0]$ & $2.8[2.0,3.0]$ & $2.9[2.6,3.0]$ & 0.12 \\
\hline Maximum score, median (IQR) & $3.0[3.0,4.0]$ & $3.0[3.0,4.0]$ & $3.0[3.0,4.0]$ & 0.007 \\
\hline Maximum score, mean $(\mathrm{SD})^{\S}$ & $3.2(0.6)$ & $3.1(0.8)$ & $3.3(0.6)$ & 0.001 \\
\hline \multicolumn{5}{|l|}{ Biochemistry (mean), median (IQR) } \\
\hline $\mathrm{pH}$ & $7.39[7.33,7.43]$ & $7.40[7.36,7.43]$ & $7.41[7.37,7.44]$ & $<0.001$ \\
\hline Potassium, mmol/L & $4.3[4.1,4.6]$ & $4.2[4.0,4.4]$ & $4.3[4.1,4.5]$ & 0.015 \\
\hline $\mathrm{BUN}, \mathrm{mmol} / \mathrm{L}^{\dagger \dagger}$ & $14.0[10.7,19.1]$ & $14.2[11.0,19.2]$ & $14.8[11.0,19.6]$ & 0.500 \\
\hline
\end{tabular}


Table 2 (continued)

\begin{tabular}{|c|c|c|c|c|}
\hline Characteristic & $\begin{array}{l}\text { Albumin } \\
4 \% \text { only }\end{array}$ & $\begin{array}{l}\text { Albumin } \\
20 \% \text { only }\end{array}$ & $\begin{array}{l}\text { Combined } \\
4 \% \text { and } 20 \%\end{array}$ & $p$ value \\
\hline \multicolumn{5}{|c|}{ Continuous RRT filters, median (IQR) } \\
\hline Average filters per day & $0.8[0.5,1.0]$ & $1.0[0.7,1.3]$ & $0.9[0.6,1.3]$ & 0.001 \\
\hline Total filters & $3.0[2.0,6.0]$ & $5.0[3.0,8.0]$ & $6.0[3.0,11.0]$ & $<0.001$ \\
\hline \multicolumn{5}{|c|}{ Continuous RRT vascular access, median [IQR] } \\
\hline Average Vascaths per day & $0.1[0.1,0.3]$ & $0.1[0.1,0.2]$ & $0.1[0.1,0.2]$ & $<0.001$ \\
\hline Total Vascaths & $1.0[1.0,1.0]$ & $1.0[1.0,2.0]$ & $1.0[1.0,2.0]$ & $<0.001$ \\
\hline
\end{tabular}

BUN, blood urea nitrogen; SOFA, sequential organ failure assessment; IQR, interquartile range; RRT, renal replacement therapy. ${ }^{\diamond}$ Refers to the albumin content of fresh frozen plasma. ${ }^{\S}$ Cohort mean of the maximum cardiovascular/respiratory SOFA score value per subject per day, included to reflect direction of association. ${ }^{\dagger \dagger}$ To convert the values for BUN to milligrammes per decilitre, divide by 0.357 . ${ }^{\ddagger}$ Displayed in online suppl. Figure 3 of Supplementary Material 1.

Table 3. Unadjusted patient outcomes of $4 \%$ versus $20 \%$ versus combined $(4+20 \%)$ albumin

\begin{tabular}{|c|c|c|c|c|}
\hline Characteristic & $\begin{array}{l}\text { Albumin } \\
4 \% \text { only }\end{array}$ & $\begin{array}{l}\text { Albumin } \\
20 \% \text { only }\end{array}$ & $\begin{array}{l}\text { Combined } \\
4 \% \text { and } 20 \%\end{array}$ & $p$ value \\
\hline Number (unless indicated, $N$ ) & $345(45)$ & $155(20)$ & $271(35)$ & \\
\hline \multicolumn{5}{|l|}{ Mortality, $n(\%)$} \\
\hline Day 28 & $148(43)$ & $68(44)$ & $108(40)$ & 0.65 \\
\hline Day 90 & $167(48)$ & $80(52)$ & $139(51)$ & 0.71 \\
\hline \multicolumn{5}{|l|}{ RRT } \\
\hline Days at day 28, med (IQR) & $4[3,10]$ & $6[4,12]$ & $9[5,20]$ & $<0.001$ \\
\hline Days at day 90, med (IQR) & $4[3,10]$ & $6[4,12]$ & $9[5,20]$ & $<0.001$ \\
\hline \multicolumn{5}{|l|}{ RRT dependence among survivors at } \\
\hline Day $28, n / N(\%)$ & $36 / 197(18)$ & $18 / 87(21)$ & $30 / 163(18)$ & 0.880 \\
\hline Day $90, n / N(\%)$ & $12 / 178(6.7)$ & $4 / 75(5.3)$ & $5 / 132(3.8)$ & 0.585 \\
\hline \multicolumn{5}{|l|}{ MV } \\
\hline Days at day 28, med (IQR) & $6[2,10]$ & $6[2,12]$ & $12[6,20]$ & $<0.001$ \\
\hline \multicolumn{5}{|l|}{ Dependence among survivors at } \\
\hline Day $28, n / N(\%)$ & $12 / 197(6.1)$ & $4 / 87(4.6)$ & $24 / 163(14.7)$ & 0.007 \\
\hline \multicolumn{5}{|l|}{ Days of stay at day 90, med (IQR) } \\
\hline In ICU from randomization & $7[3,13]$ & $9[4.5,16]$ & $16[9,27]$ & $<0.001$ \\
\hline In hospital from randomization & $15[5,33]$ & $19[9,40]$ & $28[13,54]$ & $<0.001$ \\
\hline
\end{tabular}

MV, mechanical ventilation; IQR, interquartile range; RRT, renal replacement therapy.

ated with longer duration of ICU stay. However, it was associated with longer duration of hospital stay (SHR = 0.66, 95\% CI: 0.49-0.89; $p=0.007$ ) (Table 4).

\section{Discussion}

\section{Key Findings}

We studied the administration of hyperoncotic (20\%) HAS in patients with severe AKI who were treated with continuous RRT in the RENAL trial. We found that half of RENAL trial patients received albumin therapy, and that among these, half were exposed to $20 \%$ HAS either alone or in combination with $4 \%$ HAS. A greater proportion of patients who received $20 \%$ HAS had severe organ oedema and sepsis. Patients who received only $20 \%$ HAS had a more negative mean daily fluid balance. On propensity analysis with SIPW adjustment, the administration of $20 \%$ HAS was not associated with mortality, duration of RRT, MV, or ICU length of stay. 
Table 4. Patient outcomes at day 90 , unadjusted $(n=772)$ and inverse probability of treatment weighted $(n=662)$, for administration of any $20 \%$ versus only $4 \%$ albumin

\begin{tabular}{llc}
\hline Mortality & OR $(95 \%$ CI $)$ & $p$ value \\
\hline $\begin{array}{l}\text { Death at day 90 } \\
\quad \text { Unadjusted }\end{array}$ & $1.13(0.83,1.53)$ & 0.44 \\
$\quad$ Adjusted & $1.12(0.77,1.62)$ & 0.55 \\
\hline Recovery to independence from & SHR $(95 \%$ CI $)$ & $p$ value \\
\hline RRT & $1.04(0.87,1.25)$ & 0.66 \\
$\quad$ Unadjusted & $1.04(0.84,1.30)$ & 0.70 \\
$\quad$ Adjusted & $0.92(0.75,1.12)$ & 0.39 \\
Invasive MV & $0.88(0.67,1.16)$ & 0.37 \\
$\quad$ Unadjusted & & \\
$\quad$ Adjusted & $0.82(0.70,0.96)$ & 0.01 \\
ICU admission episode & $0.81(0.63,1.04)$ & 0.11 \\
$\quad$ Unadjusted & & \\
$\quad$ Adjusted & $0.69(0.56,0.83)$ & $<0.001$ \\
Hospital admission episode & $0.66(0.49,0.89)$ & 0.007 \\
$\quad$ Unadjusted & Adjusted &
\end{tabular}

SHR, sub-hazard ratio; OR, odds ratio; CI, confidence interval; ICU, intensive care unit; MV, mechanical ventilation; RRT, renal replacement therapy.

\section{Relationship with Previous Studies}

No previous studies have investigated the association between hyperoncotic HAS and patient outcomes in patients with severe AKI, despite theoretical concerns that hyperoncotic HAS may decrease the glomerular filtration rate [14]. Previous trials such as the SAFE, ALBIOS, and CRISTAL trials compared $4 \%$ HAS to $0.9 \%$ sodium chloride, 20\% HAS to crystalloids, and various colloids to crystalloids, respectively [24-26]. All these studies failed to compare iso-oncotic $4 \%$ HAS to hyperoncotic $20 \%$ HAS and did not focus on patients with severe kidney injury receiving RRT. This is problematic as patients on RRT may be those most vulnerable to the potentially adverse effects of hyperoncotic HAS. Moreover, both 4\% HAS and 20\% HAS have mechanisms through which they may worsen AKI. For example, 4\% HAS contains a supraphysiological amount of chloride $(128 \mathrm{mmol} / \mathrm{L})$ which may cause hyperchloraemia and decrease renal cortical perfusion $[5,6,16,27,28]$. Alternatively, $20 \%$ HAS is hyperoncotic and thus may reduce glomerular net filtration pressure and therefore reduce GFR $[11,15]$. Albumin administration may also promote pro-inflammatory gene expression and increase interstitial fluid volumes with corresponding intracellular dehydration, and these ef- fects may be greatest with the higher concentration $20 \%$ HAS $[29,30]$.

The use of $20 \%$ HAS carries potential advantages. It is salt poor and should minimize the fluid volume, sodium, and chloride administered compared to $4 \%$ albumin, or indeed any other fluid [31]. These reductions may be of greater clinical significance in patients with severe AKI, who are prone to fluid overload and electrolyte derangement. In this regard, the SWIPE trial randomized $321 \mathrm{pa}-$ tients to fluid resuscitation with either $20 \%$ or $4-5 \%$ albumin within $48 \mathrm{~h}$ of admission to ICU [32]. While the SWIPE trial reported no difference in the rates of new AKI or need for RRT among patients treated with $20 \%$ HAS, it only studied 24 patients with AKI at baseline and therefore could not yield robust conclusions regarding its use in such patients. As such, ours is the first study to address this issue in hundreds of patients with severe AKI.

We previously found that HAS is often administered to more severely ill RRT patients and, after adjustment, was associated with prolonged weaning from MV and with prolonged ICU and hospital lengths of stay [22]. Our current analysis of the differential effects of HAS concentration provides a more detailed understanding of the use of albumin in patients receiving RRT for AKI and supports the safety of $20 \%$ HAS administration, forming the epidemiological basis for future controlled trials.

\section{Implications of Study Findings}

Our findings imply that, in the RENAL trial, the administration of $20 \%$ HAS was common. They also imply that oedematous patients and those with sepsis are more likely to be exposed to hyperoncotic albumin during their ICU admission. Moreover, in this context, they imply that $20 \%$ HAS therapy was associated with a more negative daily fluid balance and had no independent association with increased risk of mortality or prolongation of RRT use.

\section{Strengths and Limitations}

Our study has several strengths. As a post hoc analysis of a large, multicentre randomized trial, it used data that were prospectively collected at baseline and during treatment, and monitored for accuracy. This provided highquality, detailed and novel data regarding the administration of hyperoncotic HAS to patients requiring RRT for AKI and their subsequent outcomes. To our knowledge, this represents the largest study to provide a direct comparison of hyperoncotic to iso-oncotic albumin in critically ill patients, and the first to do so specifically in a cohort of patients with severe AKI treated with RRT. Final- 


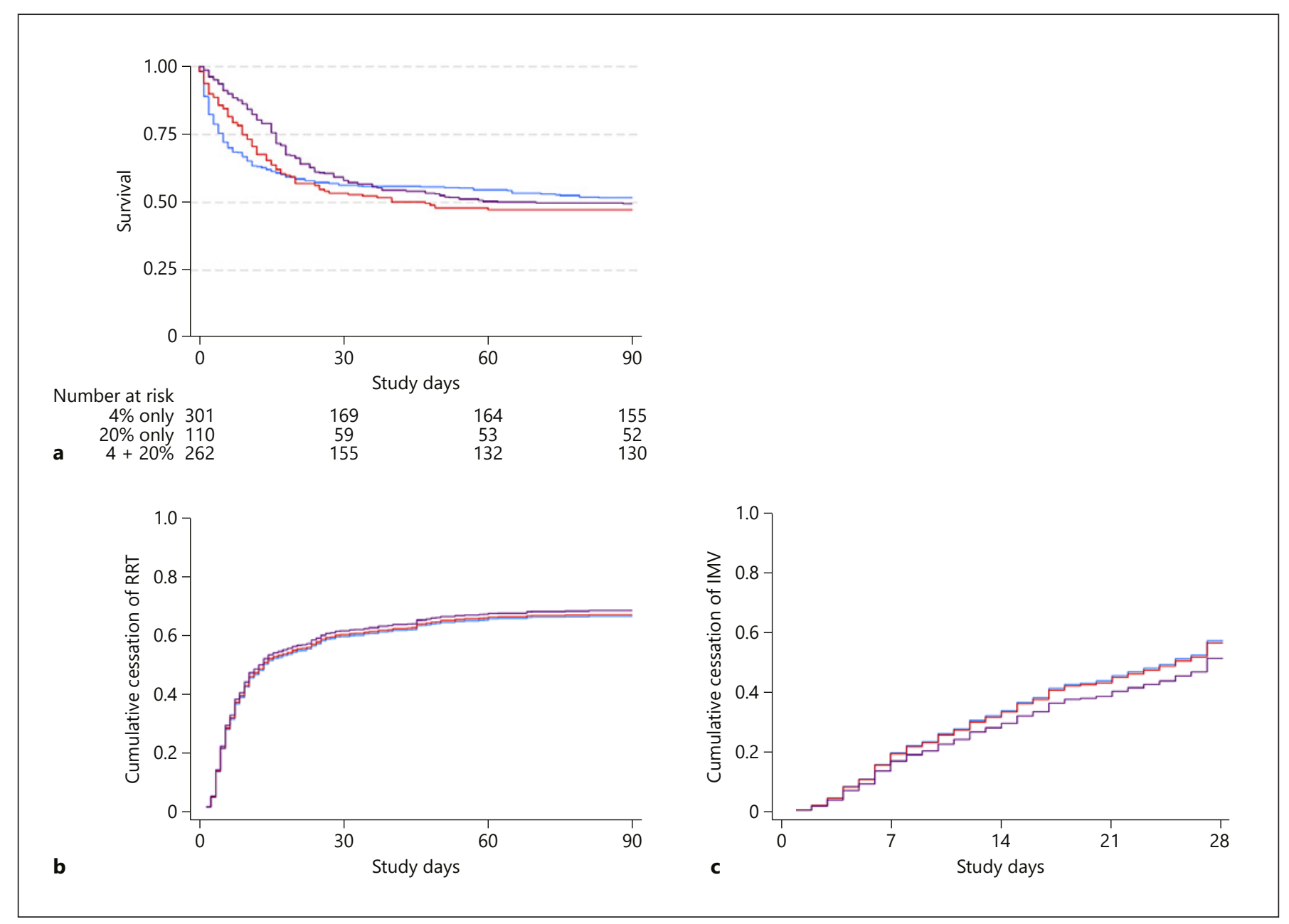

Fig. 1. Cox proportional hazards regression for death at day $90(\mathbf{a})(p=0.43)$ and competing risks regression for cessation of RRT (b) $(p=0.90)$ and $\mathrm{MV}(\mathbf{c})(p=0.51)$, stratified by albumin solution therapy concentration; $4 \%$ albumin (blue), 20\% albumin (red), both 4 and 20\% albumin (purple). All models were SIPW-adjusted. RRT, renal replacement therapy; SIPW, stabilized inverse probability of treatment weighting; MV, mechanical ventilation.

ly, we have extensively adjusted for baseline confounding in the association between 20\% HAS exposure and clinical outcomes.

We acknowledge several limitations. Our study is not a randomized controlled trial. Therefore, we can only describe associations after adjustment for measured relevant confounders. Thus, any inferences of causality are limited by the risk of unmeasured confounding. We are also unable to determine the mechanism through which associations arise. For example, the administration of hyperoncotic albumin was associated with a more negative daily fluid balance, which may reflect that these patients were at a different stage of their illness and resuscitation, or may have been because a greater proportion of these patients had severe organ oedema at baseline. Similarly, patients who were administered both $4 \%$ and $20 \%$ albumin received more packed red blood cells, which may have been in response to a haemodilutional effect of HAS or may reflect the generally greater severity of illness of these patients. Furthermore, we are unable to identify a mechanism through which hyperoncotic albumin may cause a longer hospital length of stay without altering ICU length of stay or mortality. This likely reflects unmeasured confounding, whereby clinicians chose to administer hyperoncotic albumin to more severely unwell patients who were at a higher risk of prolonged hospital stay.

The RENAL trial was conducted in Australia and New Zealand, where albumin is the colloid fluid of choice. 
Therefore, albumin may have been administered more liberally than elsewhere. This may limit the generalizability of our findings to other contexts. However, such increased use provided our study with greater statistical power because of the number of patients exposed to hyperoncotic albumin solution. The RENAL trial was conducted from 2005 to 2008 and IV fluid practises may have changed since that time. However, no data exist to confirm or refute this possibility. Unfortunately, we do not have data regarding fluid administration prior to randomization in the RENAL trial, and this is a potential confounder which may have influenced the decisions of treating clinicians. As all patients received RRT throughout the RENAL study, detailed data regarding their renal function was not available, as serum creatinine only represented the intensity of RRT. However, we used the time to cessation of RRT as an imperfect but clinically relevant patient outcome and found no association between hyperoncotic HAS therapy and the rate of recovery to RRT independence.

\section{Conclusions}

HAS was administered to half of the patients treated with continuous RRT in the RENAL trial, with half of such patients being exposed to hyperoncotic (20\%) albumin. Such $20 \%$ HAS therapy was associated with a more negative mean fluid balance and, after adjustment, was not independently associated with increased mortality or decreased rate of recovery to RRT independence. Our results support that in high-risk critically ill patients receiving RRT, 20\% HAS therapy is safe, and justify the conduct of further investigations of such therapy in this context.

\section{Statement of Ethics}

The Human Research Ethics Committees of the University of Sydney and all participating institutions approved the study (GIRE-ARF001-40-R). Written informed consent was obtained from patients or their responsible surrogate.

\section{Conflict of Interest Statement}

Professor Rinaldo Bellomo received consulting fees as an advisor for Gambro and consulted for Gambro, Biosite, Abbott, and Philipps. Dr. Gallagher received honoraria/speaking fees from Roche. The remaining authors have no conflicts of interest to declare.

\section{Funding Sources}

The RENAL study was supported by grants from the National Health and Medical Research Council (NHMRC) of Australia (grant no. 352550) and Health Research Council (HRC) of New Zealand (grant no. 06-357). The study sponsors had no role in study design; collection, analysis, and interpretation of data; writing the report; or the decision to submit the report for publication. The George Institute has received research funding from Servier, Novartis, Eisai, Merck, Sharp \& Dohme, Pfizer Australia, Fresenius Kabi Deutschland GmbH, and Sanofi Aventis. The Randomized Evaluation of Normal versus Augmented Level (RENAL) Replacement Therapy Study is a collaboration of the Australian and New Zealand Intensive Care Society Clinical Trials Group (ANZICS CTG) and the George Institute for International Health.

\section{Author Contributions}

Zachary O’Brien and Mark Finnis: conceptualization, data analysis and interpretation, drafting the manuscript, and revising the manuscript. Martin Gallagher: conceptualization, data interpretation, and revising the manuscript. Rinaldo Bellomo: conceptualization, data interpretation, drafting the manuscript, and revising the manuscript.

\section{References}

1 Finfer S, Myburgh J, Bellomo R. Intravenous fluid therapy in critically ill adults. Nat Rev Nephrol. 2018;14(9):541.

2 Finfer S, Liu B, Taylor C, Bellomo R, Billot L, Cook D, et al. Resuscitation fluid use in critically ill adults: an international cross-sectional study in 391 intensive care units. Crit Care. 2010;14(5):R185.

3 Myburgh JA, Mythen MG. Resuscitation fluids. N Engl J Med. 2013;369(13):1243-51.

4 van Haren F. Personalised fluid resuscitation in the ICU: still a fluid concept? Crit Care. 2017;21(Suppl 3):313.

5 Bellomo R, Hegarty C, Story D, Ho L, Bailey $\mathrm{M}$. Association between a chloride-liberal vs chloride-restrictive intravenous fluid administration strategy and kidney injury in critically ill adults. JAMA. 2012;308:156672.

6 Bellomo R, Glassford N, Sutcliffe H, Lam Q, Bailey M. Chloride-liberal vs. chloriderestrictive intravenous fluid administration and acute kidney injury: an extended analysis. Intensive Care Med. 2015;41:25764.

7 Myburgh JA, Finfer S, Bellomo R, Billot L, Cass A, Gattas D, et al. Hydroxyethyl starch or saline for fluid resuscitation in intensive care. N Engl J Med. 2012;367(20): 1901-11.

8 Semler MW, Self WH, Wanderer JP, Ehrenfeld JM, Wang L, Byrne DW, et al. Balanced crystalloids versus saline in critically ill adults. N Engl J Med. 2018;378(9):829-39.
9 SAFE Study Investigators. Saline or albumin for fluid resuscitation in patients with traumatic brain injury. N Engl J Med. 2007;357:874-84.

10 Perner A, Haase N, Guttormsen AB, Tenhunen J, Klemenzson G, Åneman A, et al. Hydroxyethyl starch 130/0.42 versus Ringer's acetate in severe sepsis. N Engl J Med. 2012; 367(2):124-34.

11 Wiedermann CJ, Dunzendorfer S, Gaioni LU, Zaraca F, Joannidis M. Hyperoncotic colloids and acute kidney injury: a meta-analysis of randomized trials. Critical Care. 2010;14(5): R191.

12 Schortgen F, Girou E, Deye N, Brochard L, Group CS. The risk associated with hyperoncotic colloids in patients with shock. Intensive Care Med. 2008;34(12):2157. 
13 Gomez H, Priyanka P, Bataineh A, Keener CM, Clermont G, Kellum JA. Effects of 5\% albumin plus saline versus saline alone on outcomes from large-volume resuscitation in critically ill patients. Critical Care Med. 2020; 49:79-90.

14 Hryciw N, Joannidis M, Hiremath S, Callum J, Clark EG. Intravenous albumin for mitigating hypotension and augmenting ultrafiltration during kidney replacement therapy. Clin J Am Soc Nephrol. 2020;16:8208.

15 Boer WH, Koomans HA, Dorhout Mees EJ. Renal haemodynamics and sodium handling after hyperoncotic albumin infusion in sodium-restricted normal man. Eur J Clin Invest. 1987;17(5):442-7.

16 Krajewski M, Raghunathan K, Paluszkiewicz S, Schermer C, Shaw A. Meta-analysis of high-versus low-chloride content in perioperative and critical care fluid resuscitation. $\mathrm{Br}$ J Surg. 2015;102:24-36.

17 RENAL Replacement Therapy Study Investigators. Intensity of continuous renal-replacement therapy in critically ill patients. New Engl J Med. 2009;361:162738.

18 Finfer S, Bellomo R, Cass A, Gallagher M, Lee J, Su S. The RENAL (randomised evaluation of normal versus augmented level of replacement therapy) study: statistical analysis plan. Crit Care Resusc. 2009;11:58.

19 Tamma PD, Han JH, Rock C, Harris AD, Lautenbach E, Hsu AJ, et al. Carbapenem therapy is associated with improved survival compared with piperacillin-tazobactam for patients with extended-spectrum $\beta$-lactamase bacteremia. Clin Infect Dis. 2015;60(9):131925.
20 Graham DJ, Reichman ME, Wernecke M, Hsueh YH, Izem R, Southworth MR, et al. Stroke, bleeding, and mortality risks in elderly medicare beneficiaries treated with dabigatran or rivaroxaban for nonvalvular atrial fibrillation. JAMA Intern Med. 2016;176(11): 1662-71.

21 Puri R, Nicholls SJ, Shao M, Kataoka Y, Uno K, Kapadia SR, et al. Impact of statins on serial coronary calcification during atheroma progression and regression. J Am Coll Cardiol. 2015;65(13):1273-82.

22 O'Brien Z, Finnis M, Gallagher M, Bellomo R. Early treatment with human albumin solution in continuous renal replacement patients. Blood Purif. 2020;50:205-13.

23 Austin PC, Stuart EA. Moving towards best practice when using inverse probability of treatment weighting (IPTW) using the propensity score to estimate causal treatment effects in observational studies. Stat Med. 2015; 34(28):3661-79.

24 SAFE Study Investigators. A comparison of albumin and saline for fluid resuscitation in the intensive care unit. N Engl J Med. 2004; 350:2247-56.

25 Caironi P, Tognoni G, Masson S, Fumagalli R, Pesenti A, Romero M, et al. Albumin replacement in patients with severe sepsis or septic shock. N Engl J Med. 2014;370(15):1412-21.
26 Annane D, Siami S, Jaber S, Martin C, Elatrous $\mathrm{S}$, Declère $\mathrm{AD}$, et al. Effects of fluid resuscitation with colloids vs crystalloids on mortality in critically ill patients presenting with hypovolemic shock: the CRISTAL randomized trial. JAMA. 2013;310(17):1809-17.

27 Yunos NM, Bellomo R, Story D, Kellum J. Bench-to-bedside review: chloride in critical illness. Crit Care. 2010;14:226.

28 Payen D, de Pont ACJM, Sakr Y, Spies C, Reinhart K, Vincent JL. A positive fluid balance is associated with a worse outcome in patients with acute renal failure. Crit Care. 2008;12(3): R74.

29 Wheeler DS, Giuliano JS, Lahni PM, Denenberg A, Wong HR, Zingarelli B. The immunomodulatory effects of albumin in vitro and in vivo. Adv Pharmacol Sci. 2011;2011:691928.

30 Ernest D, Belzberg AS, Dodek PM. Distribution of normal saline and 5\% albumin infusions in septic patients. Crit Care Med. 1999; 27(1):46-50.

31 Bannard-Smith J, Alexander P, Glassford N, Chan MJ, Lee M, Wong BT, et al. Haemodynamic and biochemical responses to fluid bolus therapy with human albumin solution, $4 \%$ versus $20 \%$, in critically ill adults. Crit Care Resusc. 2015;17(2):122.

32 Mårtensson J, Bihari S, Bannard-Smith J, Glassford NJ, Lloyd-Donald P, Cioccari L, et al. Small volume resuscitation with $20 \%$ albumin in intensive care: physiological effects. Intensive Care Med. 2018;44:1797-806. 